\title{
Case Study for Experience Vision - Application for PC
}

\author{
Kanako Ariya \\ Fujitsu Design Ltd. \\ Kamiodanaka 4-chome, Nakahara-ku Kawasaki, Japan 211-8588 \\ ariya@jp.fujitsu.com
}

\begin{abstract}
In order to examine the new value of photo management software preinstalled on personal computers to develop a model for the next photo management software, I have to utilize the Experience Vision method. I will introduce the process from gathering information from the activity of novice users, structuring of their real user demands, to scenario creation.
\end{abstract}

\section{Introduction}

There are many novice users among customers who purchase Fujitsu PCs. On the other hand, software developers are skilled PC users, and therefore, it is often the case that development is started without grasping beginners' actual use condition. The resulting software may be too difficult for beginners to use.

By grasping requirements in the early stage of development, improved quality and increased efficiency are expectable. The solution of those problems was tried by applying a vision centered design Experience Vision method.

\section{Process}

\subsection{Behavior Observation of Novice Users}

The project members started with collecting features and results of novice users' photograph life by observing and conducting interviews.

For this observation and interview, I prepared a "Novice Interview Template" [fig.1].

As a result, I was able to collect a series of the beginner's actions from taking a photograph, saving it to a personal computer, to arranging, editing, and printing. I was also able to obtain a lot of other notices.

\subsection{Structuration of Notices and Configuration of Personas}

Next, in order to retrieve the beginners' intrinsic value from the behavior observation that was conducted by the project members, the gathered keywords were written out to cards. 


\begin{tabular}{|c|c|}
\hline Novice Interview Template & Date: \\
\hline שUser Attributes & How do you utilize taken photographs and videos \\
\hline Age : & Print out \\
\hline Sex : & Distribute to firends \\
\hline Occupation : & Use for website/blog \\
\hline Residence : & Etc. \\
\hline \multicolumn{2}{|l|}{ Others : } \\
\hline & Dow do you save and organize photos and videos \\
\hline DC usage & Print out and paste into album \\
\hline Have my own computer Sometimes use a shared family computer & Folder management on $\mathrm{PC}$ \\
\hline \multirow[t]{2}{*}{ Do not use a computer, etc. } & Burn to DVD, etc. \\
\hline & Regarding your current usage of photos and videos, \\
\hline -Type of usually used camera & state "Would be nice to have $\cdots "$ wishes and/or episodes \\
\hline Compact digital camera Mobile phone & of successful usage etc. \\
\hline \multicolumn{2}{|l|}{ If mutliple: state main camera etc. } \\
\hline & Other things the observer noticed \\
\hline \multicolumn{2}{|l|}{ Motif, goal, timing of taking pictures } \\
\hline Taking pictures of flowers on a trip & Interview environment \\
\hline Taking pictures of grandson & PC and camera were actually used while interviewed, \\
\hline Etc. & Type of software used, etc. \\
\hline
\end{tabular}

Fig. 1. Novice Interview Template

Demands and tasks were structured depending on whether gathered information was the user's goal, or rather a mean to achieve a purpose by analyzing their relationship to each other.

Through this structure I clarified the cause and effect of problems novice users face.

Moreover, I made three personas based on the interviewed people and shared them between the project members at this time.

\subsection{Laddering Up to the Intrinsic User Value}

From the structured notices, I formed three intrinsic user values "pleasure to photograph", "pleasure to see", and "pleasure to show" based on statements, such as "I will not delete it, even if it is blurred, as long as the expression is good, ", "I want to take pictures of important events properly", "I want to make sure it is saved", These helped to create the overall structure.

\subsection{Consideration of the Advantage as a PC Maker (Business Offering Policy)}

In case of Fujitsu personal computer, the Insertion of a SD card automatically starts the importing tool.

When automation helps beginners to achieve his purpose without being confused by difficult messages and choices of the OS, it is an important aspect in the development of easy-to-use software. 
Since this interaction is a strong point unique to a PC maker who is developing both hardware and software, I decided to consider concrete scenarios based on the given interaction.

Moreover, individual users think of ease of use as an important factor in the purchase of a personal computer. When buying a personal computer, the percentage of users purchasing at a shop front of mass home electronics retailers, that ask the salesperson for an easy-to-use personal computer, is high.

Therefore, software that promise ease of use and meets the expectations when used becomes important.

\subsection{Using Structured Scenarios}

\section{Activity Scenario}

Since in the case of this example the target for the development was clear, I started from the activity scenario.

For each of the three persons' persona, usage scenes of their photo life from taking a picture to printing and the corresponding actions taken were described as an activity scenario [fig. 2].

\section{Interaction Scenario}

To some activity scenarios, I did the breakdown how things can be realized and created a detailed interaction scenario [fig. 3].

I then evaluated if the flows suit our persona, and prioritized depending on the user, and chose the scenario for prototyping.

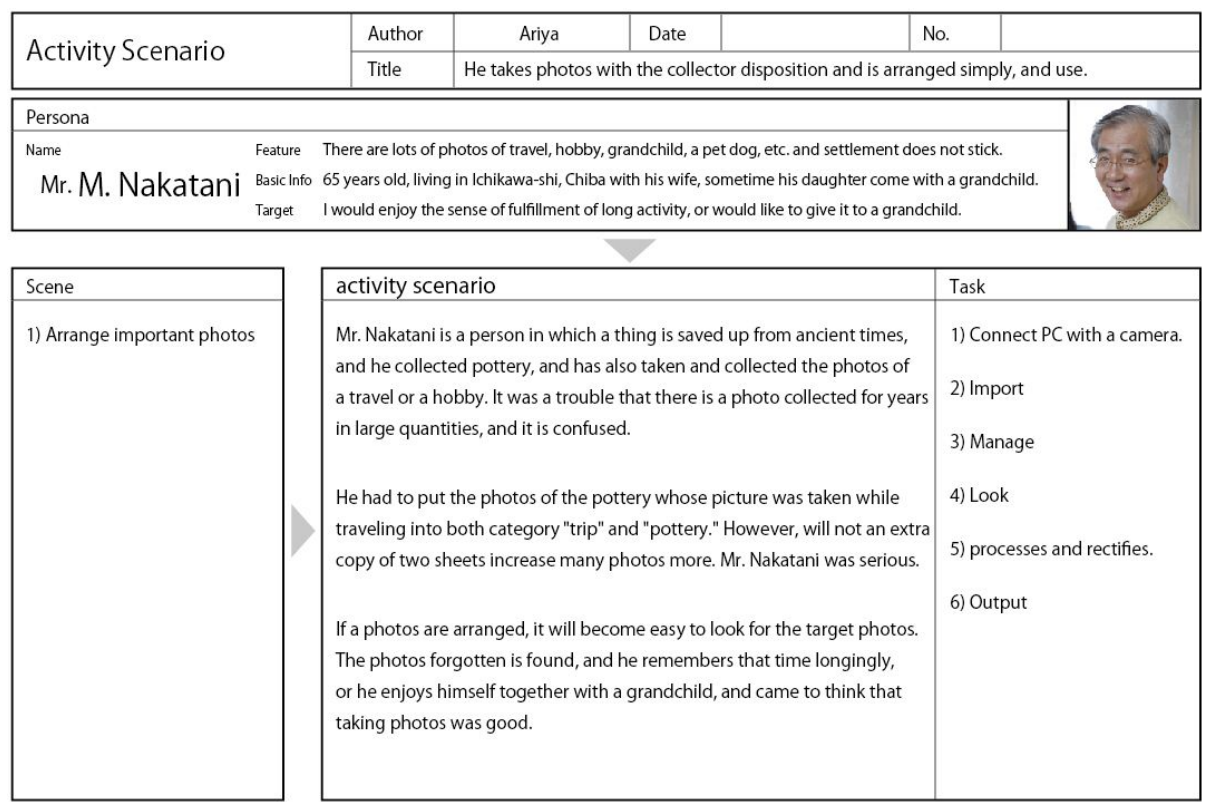

Fig. 2. Activity Scenario 


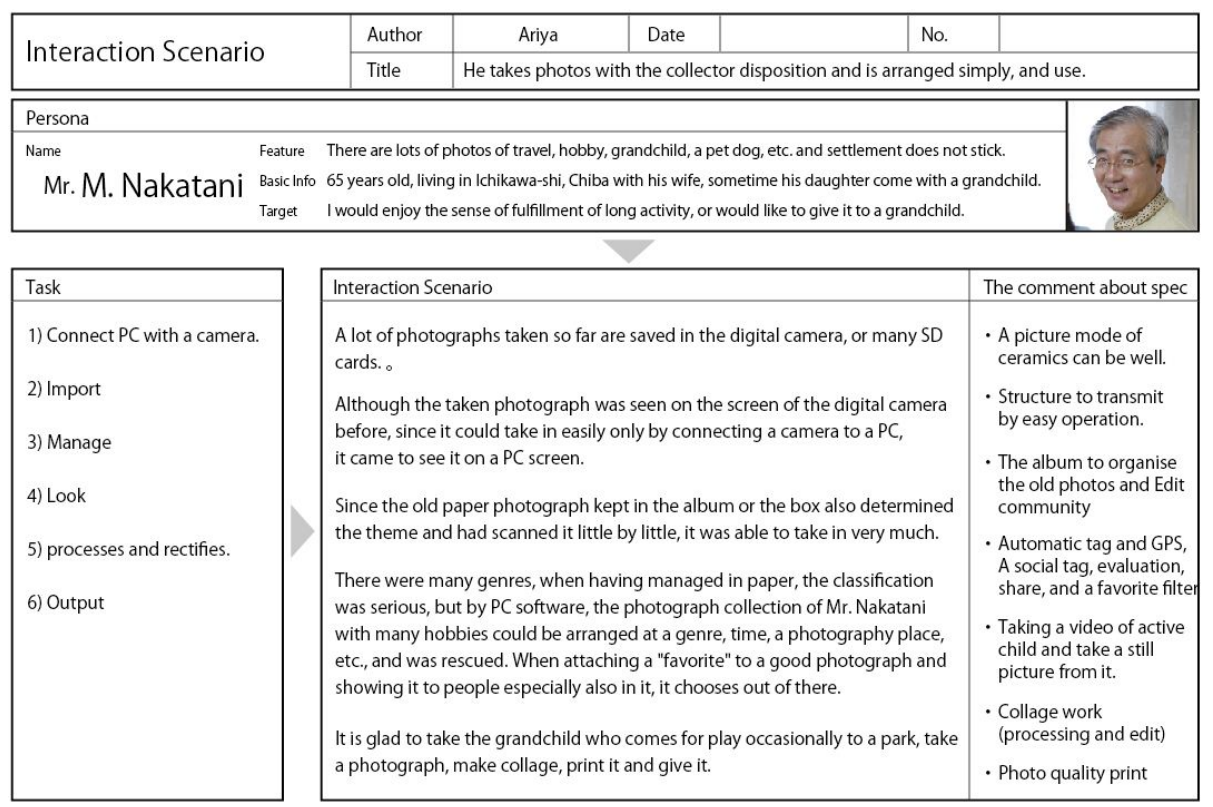

\section{Fig. 3. Interaction Scenario}

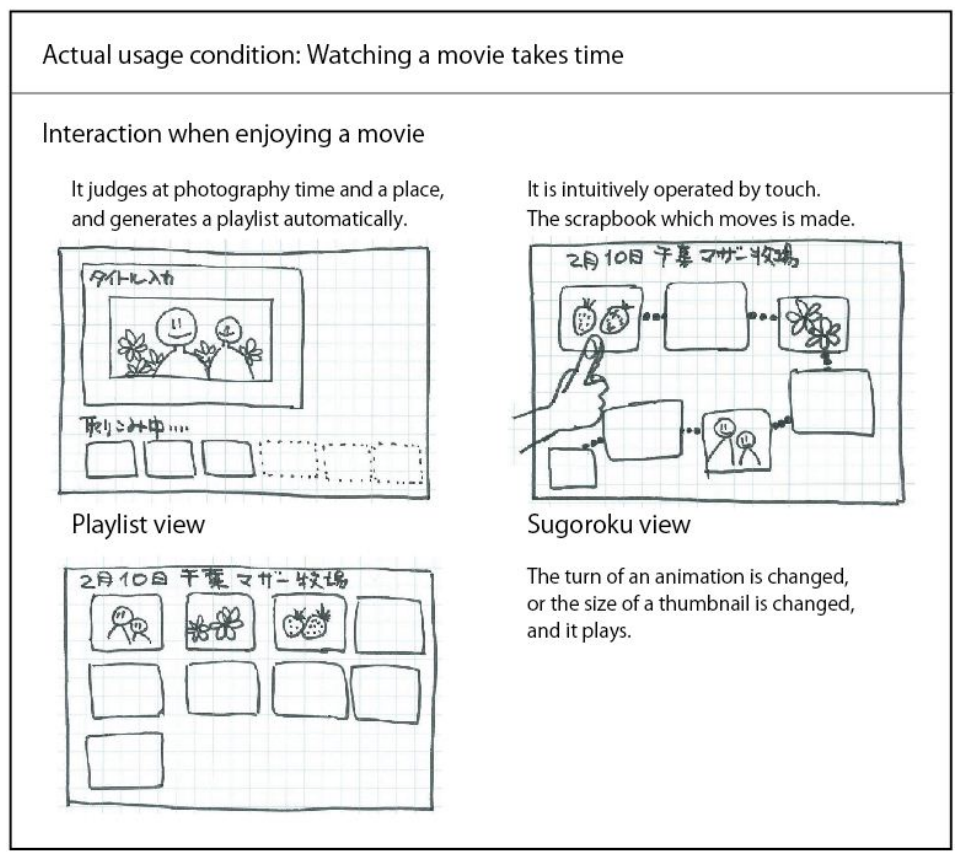

Fig. 4. User Interface Ideas 


\section{Prototyping}

From these work I extracted the user requirements for photograph software, developed the user interface design based on it, and created prototype ideas for user interface [fig. 4], [fig. 5].

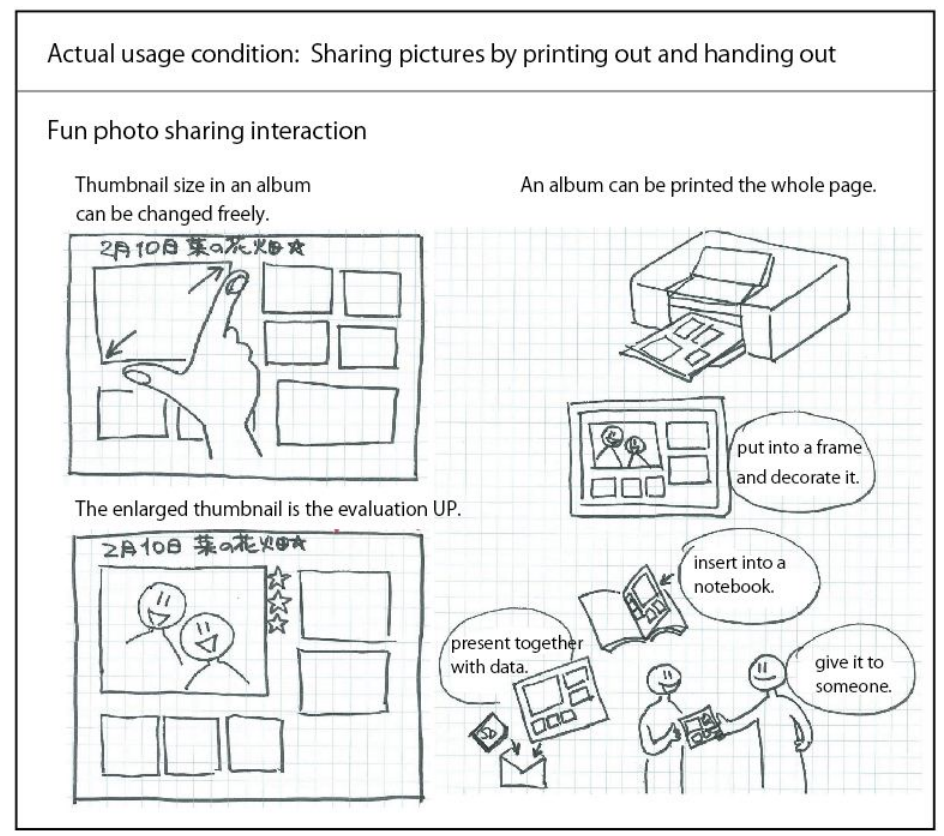

Fig. 5. User Interface Ideas

\section{Conclusion}

\subsection{The Advantages for the Mounting Process}

Vision centered design method is a technique applied in the planning phase of a product, a system, or a service. However, since it also has positive side effects in the implementation phase, an example has been introduced.

- The overview of the whole user experience by visualizing the thinking process helped to discover that the photograph experience starts from "taking a picture". A fact that I did not consciously consider in the development of previous photograph software

Since the user wants to take a picture well, displaying additional information when looking at photographs for reference, such as settings the picture has been taken with or a map of the location is appreciated. This shows how the presented method has effectively helped to gain overview of the whole User Experience of photograph browsing by clarifying the user values and actions. 
- Minimization of the redo due to change of requirements

In actual development is not rare to be obliged to a change of design.

If it is only underlying technology that changes, I can respond by only making adjustments to the interaction scenario.

In commercialization, even if functionality's implementation does not make it into the product due to deadlines, keeping the results of the examination process, helps to not forget the ideal design which can be utilized for the next development cycle.

\subsection{Problems of the Execution}

When utilizing the presented Experience Vision method I noticed the need for the following points in practical use.

- Dividing into teams

Taking photos, seeing photos, sharing photos - By dividing into teams for each activity, discussions and scenario creation become more efficient.

Moreover, by having two or more teams competing each other examine the same activity, substantial scenario creation can be done even if only a short period of time is given.

- Raising facilitators

During discussions considering of business viewpoints may occur. As business viewpoints may sometimes differ from the user viewpoint, it is important to raise a reliable facilitator who can cope with such situations appropriately.

- Rooting the value creation process

For successful examination of this method it is important to be useful for the improvement of a product, and that all stakeholders of the development process to understand that the method helps to guide a product to business success, and that the method is integrated into the development process. Therefore, the development related members also need to change their mind to successfully adopt the presented method. 